\title{
ПАТОГЕНЕЗ И МЕТОДЫ ЛЕЧЕНИЯ ИНФЕРТИЛЬНОСТИ ПРИ ЭНДОМЕТРИОЗЕ (ОБЗОР ЛИТЕРАТУРЫ)
}

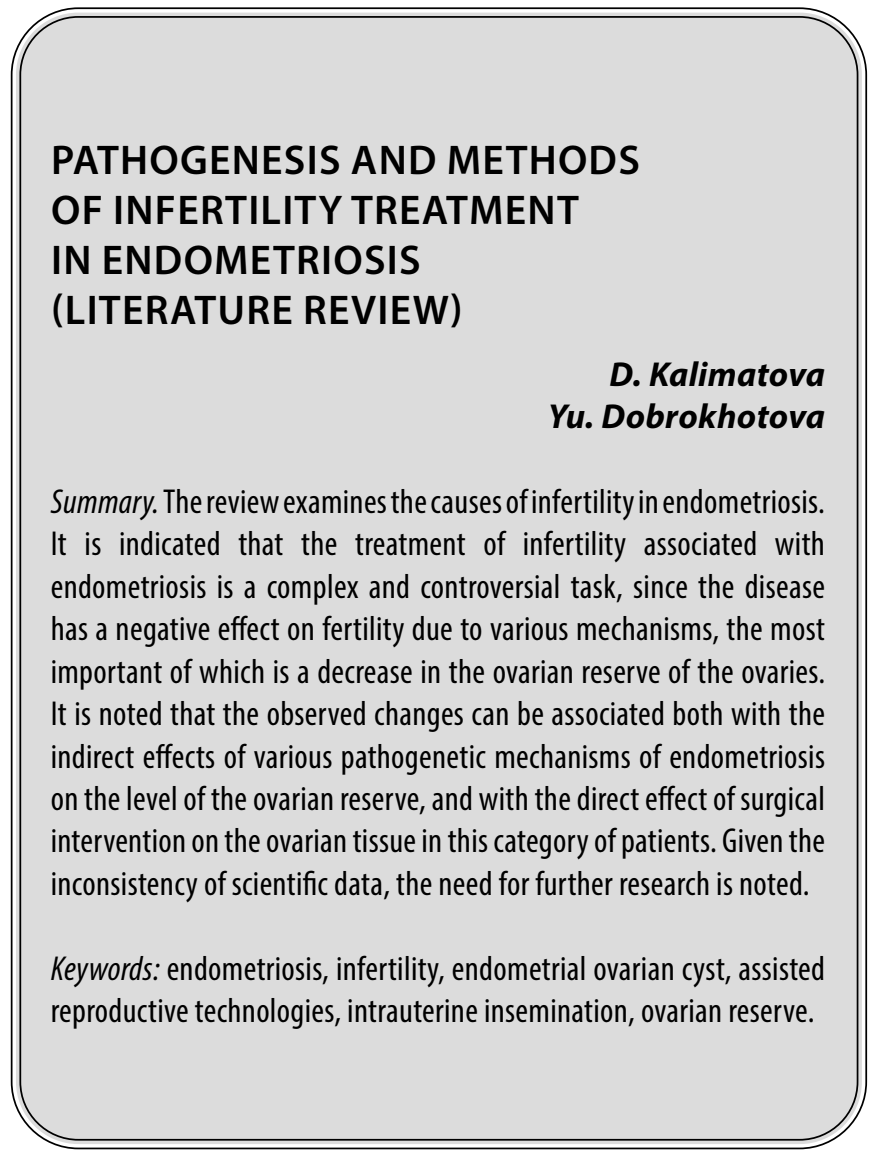

\section{Введение}

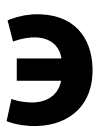
ндометриоз представляет собой комплексный синдром, в основе патогенеза и клинический проявлений которого лежит эстрогензависимый хронический воспалительный процесс, который поражает преимущественно тазовые органы, в первую очередь - яичники [1, 2]. Заболевание является наиболее распространенной причиной хронической тазовой боли и одним из важнейших факторов бесплодия $[2,3]$. У $25-50 \%$ женщин с бесплодием диагностируется эндометриоз, и приблизительно 30-50\% пациенток с эндометриозом страдают от бесплодия. Частота встречаемости эндометриоза среди всех пациенток, прибегающих к вспомогательным репродуктивным технологиям (ВРТ), составляет в среднем 20-35\% [3-5].

Одним из дискуссионных вопросов до настоящего времени остается необходимость хирургического лечения эндометриоза у пациенток с бесплодием. По дан-
Калиматова Донна Магомедовна

К.м.н., ФГБОУ ВО «Российский национальный исследовательский медицинский университет имени Н.И. Пирогова» Министерства здравоохранения Российской Федерации 9227707@gmail.com

Доброхотова Юлия Эдуардовна Д.м.н., профессор, ФГБОУ ВО «Российский национальный исследовательский медицинский университет имени Н.И. Пирогова» Министерства здравоохранения Российской Федерачии

Аннотация. В обзоре рассмотрены причины бесплодия при эндометриозе. Указано, что лечение бесплодия, связанного с эндометриозом, является сложной и неоднозначной задачей, поскольку заболевание оказывает негативное влияние на фертильность за счет различных механизмов, важнейшим из которых является снижение овариального резерва яичников. Отмечено, что наблюдаемые изменения могут связаны как с опосредованным влияниям различных патогенетических механизмов эндометриоза на уровень овариального резерва, так и с непосредственным воздействием хирургического вмешательства на ткань яичников у данной категории пациенток. Учитывая противоречивость научных данных, отмечена необходимость дальнейших исследований.

Ключевые слова: эндометриоз, бесплодие, эндометриальная киста яичника, вспомогательные репродуктивные технологии, внутриматочная инсеминация, овариальный резерв.

ным ряда исследований, операция может привести к повреждению ткани яичника и снижению овариального резерва (OP) $[6,7]$. В связи с этим сегодня перед гинекологами и репродуктологами стоят важные задачи: обеспечить минимальную травматичность хирургических вмешательств на яичниках и оценить их функциональное состояние в послеоперационном периоде. Таким образом, ведение пациенток с бесплодием и эндометриозом - сложная и актуальная задача, требующая поиска эффективных методов ее решения.

\section{Шель работы}

Анализ литературных данных о современных подходах в лечению бесплодия у женщин с эндометриозом.

Термин «эндометриоз» изначально описывал гистологический диагноз, однако по мере формирования представлений о клинической картине этой патологии стал применяться для описания симптомокомплекса 
поражения различных тканей в области таза. С анатомической точки зрения тазовый эндометриоз характеризуется наличием эндометриальной ткани за пределами полости матки, как правило, в тазовой брюшине, яичниках или других органах (в том числе в кишечнике) $[2,5]$.

Как показали результаты исследований, фертильность при эндометриозе снижается в результате развития резистентности эндометрия к прогестерону, усилению пролиферации и подавлению апоптоза, которые, в свою очередь, вызваны дисрегуляцией сигнальных путей рецептивности эндометрия, которая наблюдается при эндометриозе $[3,8]$.

Причинная связь между эндометриозом и бесплодием была подтверждена результатами ряда рандомизированных клинических исследований (РКИ), проведенных на основании принципов доказательной медицины. Так, в крупном когортном исследовании, в которое были включены женщины моложе 35 лет, было показано, что при наличии эндометриоза риск бесплодия в два раза выше, чем у женщин без признаков заболевания [9]. Ежемесячный коэффициент рождаемости у пациенток с эндометриозом составил 2-10\% по сравнению с 15-20\% у здоровых женщин, при этом было показано, что уровень данного показателя обратно пропорционален тяжести эндометриоза. При легком эндометриозе зачатие без каких-либо вмешательств возможно примерно в 50\% случаев, при эндометриозе средней тяжести - в 25\% случаев, при тяжелой форме заболевания спонтанное зачатие происходит в единичных случаях [10].

В ряде исследований показано, что поражение тканей яичников при эндометриозе негативно сказывается на их функции, нарушает работу естественных репродуктивных механизмов [4, 11-13]. Установлено, что эндометриоз и ассоциированные с ним структурные изменения коры яичников снижают ОР $[14,15]$. Предложено несколько потенциальных механизмов повреждения тканей яичников при этом заболевании. Так, показано, что растущая эндометриальная киста яичника (ЭКЯ) может механически повреждать его ткань, в том числе фолликулы; кроме того, кисты нарушают микроциркуляцию в органе за счет сдавления окружающей коры яичников [5].

Предполагается также, что важную роль в повреждении ткани яичников играет воспалительная реакция при эндометриозе. Кистозная жидкость в больших концентрациях содержит токсические вещества, в частности, активные формы кислорода (АФК), протеолитические ферменты и свободное железо $[12,15]$. В экспериментальных моделях было показано, что со- держимое ЭКЯ негативно влияет на рост фолликулов [5]. Продемонстрировано, что неблагоприятные эффекты в отношении прилежащих к очагам эндометриоза тканей обусловлено изменениями экспрессии цитокинов, снижением уровней тестостерона и антимюллерова гормона (АМГ), а также нарушениями липидного и белкового обмена в ткани яичника $[2,12]$.

Продемонстрировано, что сресс, вызванный АФК, вызывает апоптоз яйцеклеток и некроз фолликулов на ранних стадиях их развития. Более того, АФК вместе с трансформирующим фактором роста бета индуцируют фиброз тканей, сопровождающийся утратой фолликулов. Хронический фиброз вызывает прогрессирующее снижение фолликулярного резерва яичников и качества яйцеклеток [13-15].

В ряде РКИ продемонстрировано отрицательное влияние эндометриоза на ОР [10, 16-18]. Для анализа взаимосвязи между резервом яичников и тяжестью эндометриоза используется система American Society for Reproductive Medicine (ASRM), согласно которой заболевание подразделяется на 4 категории: минимальную (стадия I), легкую (II), среднюю (III) и тяжелую (IV).

Almog B. et al. (2011) показали, что в яичнике с неоперированными эндометриомами значение показателя количества антральных фолликулов ниже, чем в контралатеральном здоровом яичнике, чего не наблюдается при других доброкачественных кистах яичников [16]. Kim J.Y. et al. (2013) сравнили две группы сопоставимых по возрастному составу и индексу массы тела женщин, которым выполнялись резекции эндометриом либо зрелых кистозных тератом яичников. Было показано, что сывороточная концентрация АМГ у женщин с тяжелым эндометриозом (IV степень по ASRM) значительно ниже, чем у женщин с тератомами [19].

Kasapoglu I. et al. (2018) оценивали, носит ли снижение ОР, обусловленное эндометриозом, прогрессирующий характер, сопоставив в своей работе скорость этого процесса с естественном снижением исследуемого показателя. Было показано, что концентрации АМГ, являющегося маркером уровня ОР, при эндометриозе снижаются быстрее, чем в контрольной группе женщин (без эндометриоза) [17].

На сегодняшний день лечение бесплодия, ассоциированного с эндометриальными кистами яичников (ЭКЯ), включает выжидательную тактику, хирургическое удаление кист и применение ВРТ, в частности, экстракорпорального оплодотворения (ЭКО) [20, 21]. Медикаментозное лечение не играет существенной роли в терапии бесплодия, однако может применяться перед планируемым ЭКО с целью улучшения его исходов. 
Несмотря на то, что риск снижения ОР в результате операции по удалению эндометриомы, очевидно, превышает таковой при дренировании или абляции кисты, обобщение данных ряда РКИ в рамках Кокрановского обзора показало, что удаление эндометриомы ассоциируется с повышением шансов наступления беременности в естественном цикле и снижением частоты рецидивов ЭКя [22]. В то же время до настоящего времени не доказано, что наличие ЭКЯ связано напрямую с высоким риском бесплодия. В доступной литературе практически отсутствуют сведения о результатах РКИ по сравнительной оценке эффективности оперативного лечения ЭКЯ и выжидательной тактики у этого контингента пациенток с бесплодием, планирующих ЭКО. Нет четких данных о том, что хирургическое удаление ЭКЯ оказывает негативное влияние на ОР: наряду с его снижением после хирургического лечения показано и частичное восстановление уровня ОР после выполненной операции $[19,20,22]$.

У пациенток с тяжелым эндометриозом (стадии III и IV по классификации ASRM) коэффициент рождаемости, вероятность клинической беременности и среднее количество яйцеклеток, получаемое в течение цикла фертилизации in vitro существенно ниже, чем у женщин без эндометриоза [18]. Показано, что индукция овуляции в сочетании с внутриматочной инсеминацией достоверно повышает риск рецидива тазовой боли и прогрессирнования заболевания, поэтому фертилизация in vitro является наиболее предпочтительным методом лечения бесплодия при эндометриозе.

Хирургические вмешательства при эндометриозе выполняются лапароскопически и лапаротомически, а также с применением методов роботизированной хирургии. Лапароскопический доступ обладает рядом преимуществ, к которым относится более короткий период реабилитации и низкая стоимость лечения. В то же время хирургическое лечение эндометриоза, как отмечено выше, сопровождается риском повреждения яичников, в связи с чем предлагаются методы минимизации повреждения тканей при выполнении вмешательства, в частности, метод «стриппинга» (вылущивание ЭКЯ), который тем не менее может приводить к повреждению непораженных тканей яичника [23].

Электрокоагуляция паренхимы яичников после удаления стенки кисты может приводить к термическому повреждению ткани органа, в результате чего происходит снижение концентрации АМГ. Повреждение тканей яичника может затрагивать сосудистые структуры, нарушая микроциркуляцию. К факторам, снижающим функцию яичников в течение первой недели после различных видов хирургических вмешательств, относятся воспаление, отек и ишемия. Наблюдаемое впоследствии долгосрочное прогрессивное снижение ОР связывают с нарушениями васкуляризации ткани яичника [24].

Raffi F. et al. (2012) проанализировали результаты 8 проспективных исследований и установили, что уровень АМГ после оперативного вмешательства значительно снизился (в среднем на 38\%). При односторонней резекции эндометриомы уровень АМГ уменьшался на 30\%, при билатеральной резекции - на 44\% [25]. Somigliana E. et al. (2012) проанализировали результаты 11 исследований, в 9 из которых авторы сообщили о значительном снижении концентрации АМГ после удаления ЭКЯ [26].

Показано, что при лечении минимального или легкого эндометриоза (степени I и II по ASRM) применение лапароскопических вмешательств с выполнением резекции или абляции эндометриоидных очагов способствует увеличению вероятности спонтанной беременности и живорождения по сравнению с использованием выжидательной тактики лечения [25].

При сравнении методов лапароскопического лечения эндометриом яичников размером более 3-4 см было выявлено, что резекция кисты превосходит по эффективности дренирование и абляцию ЭКЯ в отношении вероятности возникновения спонтанной беременности у субфертильных пациенток [23, 24].

В ряде исследования показано, что у женщин с эндометриозом и бесплодием кумулятивная вероятность беременности через 3 года после хирургического вмешательства составила 47\%, при этом вероятность беременности не зависела от стадии заболевания по ASRM. Отмечают, что частота спонтанной беременности после хирургического лечения значительно выше, чем при выжидательной тактике, при умеренном эндометриозе и тяжелом эндометриозе [27, 28]. С учетом этих результатов хирургическое лечение данной категории пациенток следует рассматривать в качестве альтернативы использованию консервативной тактики. В то же время в подобных случаях необходимо учитывать вероятность послеоперационного снижения функции яичников и информировать об этом пациенток.

Установлено, что использование биполярной коагуляции для гемостаза после резекции эндометриомы яичника снижает ОР яичника в большей степени, чем применение гемостатических швов и герметиков. Таким образом, при выполнении операции следует стремиться к минимизации термического поражения, обусловленного применением вышеуказанного метода [29]. 
При выполнении лапароскопического вмешательства следует проводить тканесберегающие операции, целью которых является сохранение OP. Так, Donnez J. et al. (2010) предложили метод, который заключается в удалении части кисты яичника, расположенной дальше всего от ворот органа, путем традиционной резекции ЭКЯ, оставшиеся 10-20\% стенки эндометриомы удаляются с помощью воздействия энергии углекислотного лазера [23].

В целом следует отметить, что к настоящему времени отсутствуют однозначные свидетельства о превосходстве того или иного метода лечения бесплодия при эндометриозе. Для минимизации негативного влияния традиционного «стриппинга» были предложены двух- и трехэтапные варианты вмешательства, в ходе которых на первом этапе выполняется дренирование кистозной жидкости, ирригация и биопсия, затем на протяжении 3 месяцев пациентка получает агонист гонадотропин-рилизинг гормона для подавления очага эндометриоза. При этом диаметр кисты уменьшается примерно на 50\%. Через 3 месяца выполняется вторая операция, в ходе которой происходит удаление стенки кисты с помощью углекислотного лазера [27].

Полученные данные свидетельствуют, что использование многоэтапного подхода позволяет избежать удаления нормальной ткани яичника и минимизировать термическое поражение за счет использования энергии лазерного излучения, благодаря чему обеспечивается сохранение ОР яичников [30].

\section{Применение вспомогательных}

репродуктивных технологий в лечении

бесп^оАия при эн Аометриозе

Внутриматочная инсеминация (ВМИ) является относительно простой процедурой по сравнению с ЭКО, ее эффективность была продемонстрирована в многочисленных исследованиях, в которые были включены пары, в которых у женщин был минимальный и легкий эндометриоз, у партнеров - нормальное качество спермы. Полагают, что у пациенток с эндометриозом I и II стадий по ASRM контролируемая стимуляция яичников (с помощью кломифена цитрата или гонадотропинов) в сочетании с ВМИ может рассматриваться в качестве альтернативы проведению только ВМИ, ЭКО или дальнейшего хирургического лечения [31].

Выполнение резекции ЭКЯ при лечении бесплодия в отличие от абляции сопровождается повышением фолликулярного ответа яичников на стимуляцию гонадотропинами. В то же время данные, однозначно подтверждающие превосходство резекции ЭКЯ по сравнению с абляцией в отношении вероятности беременности после последующих ВМИ и стимуляции яичников, отсутствуют. Необходимо отметить, что ВМИ, как правило, не выполняется при среднем и тяжелом эндометриозе в связи с высоким риском спаечного процесса и поражения фаллопиевых труб. В таких случаях следует рассмотреть возможность выполнения ЭКО.

Как отмечено выше, эндометриоз яичников сопровождается снижением ОР, в связи с чем ответ на стимуляцию гонадотропинами при данной патологии может быть снижен. Показано, что у женщин с односторонними эндометриомами яичников наблюдается снижение ответа на контролируемую гиперстимуляцию, выполняемую в рамках цикла ЭКО [26]. Ответ яичников в значительной степени зависел от размера и количества эндометриом. В то же время, по результатам ретроспективного анализа, выполненного Esinler l. et al. (2012), было показано, что в циклах ЭКО у пациенток с односторонними эндометриомами количество антральных фолликулов и общее количество полученных ооцитов были такими же, как у женщин без признаков эндометриоза. При этом не отмечено зависимости между размерами и количеством эндометриом, с одной стороны, и количеством полученных ооцитов, с другой [32].

По данным мета-анализа Hamdan M. et al. (2015), было показано, что вероятность возникновения беременности у пациенток с эндометриозом в результате ЭКО без хирургического вмешательства была такой же, как у женщин без эндометриоза, однако среднее количество полученных ооцитов у пациенток с наличием признаков заболевания ниже, а вероятность прекращения цикла была значительно выше, чем у здоровых пациенток [18].

В некоторых работах было показано, что резекция эндометриомы не оказывает отрицательного влияния на их ответ при выполнении циклов ЭКО. Benaglia L. et al. (2011) представили результаты исследования, в которое были включены 93 женщины, которым выполнялась резекция односторонней эндометриомы. У 12 пациенток, принявших участие в исследовании, в оперированном яичнике полностью отсутствовал фолликулярный рост, однако в контралатеральном яичнике процессы фолликулообразования оставались без изменений [33].

Hong S. et al. (2017) установили, что эффективность применения ВРТ у женщин со сниженным ОР была сходной как после выполнения им резекции эндометриомы, так и при отсутствии хирургических вмешательств в анамнезе. В группах этих пациенток не различались 
показатели ОР, частоты беременности и живорождения [34].

Очевидно, что при минимальном или легком эндометриозе и вызванном им бесплодии следует рассматривать возможность хирургического лечения и выполнения последующей ВМИ. При средней тяжести и тяжелом эндометриозе хирургическое лечение может приводить к снижению ОР яичников и негативно повлиять на результаты последующего ЭКО [22]. Перед принятием решения о необходимости хирургического лечения эндометриоза у пациенток репродуктивного возраста следует оценивать ОР, а также измерять уровень АМГ минимум через 3 месяца после операции для определения дальнейшей тактики лечения.

\section{Зак^ючение}

Анализ данных литературы свидетельствует, что лечение бесплодия, связанного с эндометриозом, является сложной и неоднозначной задачей, поскольку заболевание оказывает негативное влияние на фертильность за счет различных механизмов, важнейшим из которых является снижение ОР яичников. При этом наблюдаемые изменения могут связаны как с опосредованным влияниям различных патогенетических механизмов эндометриоза на уровень ОР, так и с непосредственным воздействием хирургического вмешательства на ткань яичников у данной категории пациенток.

Медикаментозная терапия эндометриоза практически не влияет на бесплодие, в то время как данных, достоверно подтверждающих оправданность хирургического вмешательства при бесплодии, ассоциированном с эндометриозом, в настоящее время недостаточно. Внутриматочная инсеминация является методом первой линии после хирургического лечения минимального или легкого эндометриоза в связи с относительной простотой ее выполнения. Для пациенток с тяжелым эндометриозом, возможно, наиболее эффективным представляется использование методов экстракорпорального оплодотворения.

С целью совершенствования подходов к лечению эндометриоза и ассоциированного с ним бесплодия актуальным представляется дальнейшее изучение патогенеза инфертильности при этом заболевании, результаты этих исследований будет способствовать углублению понимания патофизиологии эндометриоза и разработке перспективных подходов к лечению бесплодия.

\section{ЛИТЕРАТУРА}

1. Адамян Л.В., Арсланян К.Н., Сонова М.М. и др. Современные тенденции в хирургическом лечении инфильтративных форм эндометриоза. Проблемы репродукции. 2019; 25 (5): 36-41.

2. Gruber TM, Mechsner S. Pathogenesis of Endometriosis: The Origin of Pain and Subfertility. Cells. 2021 Jun 3;10(6):1381. doi: 10.3390/cells10061381.

3. Tanbo T., Fedorcsak P. Endometriosis-associated infertility: aspects of pathophysiological mechanisms and treatment options. Acta Obstet. Gynecol. Scand. 2017; 96: 659-667.

4. Bulun S.E., Yilmaz B.D., Sison C. Endometriosis. Endocrine Reviews. 2019; 40(4): 1048-1079.

5. Lee D, Kim SK, Lee JR, Jee BC. Management of endometriosis-related infertility: Considerations and treatment options. Clin Exp Reprod Med. 2020 Mar;47(1):111. doi: 10.5653/cerm.2019.02971.

6. Boujenah J., Salakos E., Pinto M. et al. Endometriosis and uterine malformations: infertility may increase severity of endometriosis. Acta 0bstet. Gynecol. Scand. 2017; 96 (6): 702-706.

7. Tomassetti C., D'Hooghe T. Endometriosis and infertility: insights into the causal link and management strategies. Best. Pract. Res. Clin. Obstet. Gynaecol. 2018; 51:25-33.

8. Filip L., Duică F., Prădatu A. et al. Endometriosis Associated Infertility: A Critical Review and Analysis on Etiopathogenesis and Therapeutic Approaches. Medicina (Kaunas). 2020 Sep 9;56(9):460. doi: 10.3390/medicina56090460.

9. Malvezzi H., Marengo E.B., Podgaec S., Piccinato C.A. Endometriosis: current challenges in modeling a multifactorial disease of unknown etiology. J. Transl. Med. 2020; 18(1): 311. doi: 10.1186/s12967-020-02471-0.

10. Prescott J., Farland L.V., Tobias D.K. et al. A prospective cohort study of endometriosis and subsequent risk of infertility. Hum. Reprod. $2016 ; 31: 1475-1482$.

11. Llarena N.C., Falcone T., Flyckt R.L. Fertility Preservation in Women With Endometriosis. Clin. Med. Insights Reprod. Health. 2019; 13:1179558119873386. doi: $10.1177 / 1179558119873386$.

12. Matsuzaki S., Schubert B. Oxidative stress status in normal ovarian cortex surrounding ovarian endometriosis. Fertil. Steril. 2010; 93: $2431-2432$.

13. Kimber-Trojnar Ż., Pilszyk A., Niebrzydowska M. et al. The Potential of Non-Invasive Biomarkers for Early Diagnosis of Asymptomatic Patients with Endometriosis. J. Clin. Med. 2021; 10 (13): 2762. doi: 10.3390/jcm10132762

14. Wang L., Tang J., Wang L. et al. Oxidative stress in oocyte aging and female reproduction. J. Cell Physiol. 2021; Jun 14. doi: 10.1002/jcp.30468.

15. Sanchez A.M., Pagliardini L., Cermisoni G.C. et al. Does Endometriosis Influence the Embryo Quality and/or Development? Insights from a Large Retrospective Matched Cohort Study. Diagnostics (Basel). 2020; 10 (2): 83. 
16. Almog B., Shehata F., Sheizaf B. et al. Effects of ovarian endometrioma on the number of oocytes retrieved for in vitro fertilization. Fertil. Steril. 2011; 95: 525-527.

17. Kasapoglu I., Ata B., Uyaniklar 0. et al. Endometrioma-related reduction in ovarian reserve (ERROR): a prospective longitudinal study. Fertil. Steril. 2018; 110: 122-127.

18. Hamdan M., Omar S.Z., Dunselman G., Cheong Y. Influence of endometriosis on assisted reproductive technology outcomes: a systematic review and metaanalysis. Obstet. Gynecol. 2015; 125 (1): 79-88.

19. Kim J.Y., Jee B.C., Suh C.S., Kim S.H. Preoperative serum anti-Mullerian hormone level in women with ovarian endometrioma and mature cystic teratoma. Yonsei Med. J. 2013; 54: 921-926.

20. de Ziegler D., Pirtea P., Carbonnel M. et al. Assisted reproduction in endometriosis. Best Pract. Res. Clin. Endocrinol. Metab. $2019 ; 3$ (1): 47-59.

21. Oliveira R., Adami F., Mafra F.A. et al. Causes of endometriosis and prevalent infertility in patients undergoing laparoscopy without achieving pregnancy. Minerva Ginecol. 2016; 68 (3): 250-258.

22. Dunselman G.A., Vermeulen N., Becker C. et al. ESHRE guideline: management of women with endometriosis. Hum. Reprod. 2014; $29: 400-412$.

23. Donnez J., Lousse J.C., Jadoul P. et al. Laparoscopic management of endometriomas using a combined technique of excisional (cystectomy) and ablative surgery. Fertil. Steril. 2010; 94: 28-32.

24. Duffy J.M., Arambage K., Correa F.J. et al. Laparoscopic surgery for endometriosis. Cochrane Database Syst. Rev. 2014;(4): CD011031.

25. Raffi F., Metwally M., Amer S. The impact of excision of ovarian endometrioma on ovarian reserve: a systematic review and meta-analysis. J. Clin. Endocrinol. Metab. 2012; 97: 3146-3154.

26. Somigliana E., Benaglia L., Paffoni A. et al. Risks of conservative management in women with ovarian endometriomas undergoing IVF. Hum. Reprod. Update. 2015; 21: 486-499.

27. Jadoul P., Kitajima M., Donnez 0. et al. Surgical treatment of ovarian endometriomas: state of the art? Fertil. Steril. 2012; 98: $556-563$.

28. Goldberg J.M., Falcone T., Diamond M.P. Current controversies in tubal disease, endometriosis, and pelvic adhesion. Fertil. Steril. 2019; 112(3):417-425. doi: 10.1016/j.fertnstert.2019.06.021.

29. Ata B., Turkgeldi E., Seyhan A., Urman B. Effect of hemostatic method on ovarian reserve following laparoscopic endometrioma excision: comparison of suture, hemostatic sealant, and bipolar dessication: a systematic review and meta-analysis. J. Minim. Invasive Gynecol. 2015; 22: 363-372.

30. Tsolakidis D., Pados G., Vavilis D. et al. The impact on ovarian reserve after laparoscopic ovarian cystectomy versus three-stage management in patients with endometriomas: a prospective randomized study. Fertil. Steril. 2010; 94: 71-77.

31. Jeon Y.E., Jung J.A., Kim H.Y. et al. Predictive factors for pregnancy during the first four intrauterine insemination cycles using gonadotropin. Gynecol. Endocrinol. 2013; 29: 834-838.

32. Esinler I., Bozdag G., Arikan I. et al. Endometrioma $\leq 3 \mathrm{~cm}$ in diameter per se does not affect ovarian reserve in intracytoplasmic sperm injection cycles. Gynecol. Obstet. Invest. 2012; 74: 261-264.

33. Benaglia L., Somigliana E., Santi G. et al. IVF and endometriosis-related symptom progression: insights from a prospective study. Hum. Reprod. 2011; 26: 368-2372.

34. Hong S.B., Lee N.R., Kim S.K. et al. In vitro fertilization outcomes in women with surgery induced diminished ovarian reserve after endometrioma operation: comparison with diminished ovarian reserve without ovarian surgery. Obstet. Gynecol. Sci. 2017; 60: 63-68.

с Калиматова Донна Магомедовна (9227707@gmail.com ), Доброхотова Юлия Эдуардовна. Журнал «Современная наука: актуальные проблемы теории и практики» 